\title{
Liderazgo del director y motivación docente en Juanjuí, San Martín - Perú
}

Leadership of the principal and teacher motivation in Juanjuí, San Martín - Peru

Liderança do diretor e motivação do professor em Juanjuí, San Martín - Peru

\author{
Renán Saavedra Sandoval \\ rsaavedrasa@unmsm.edu.pe \\ Escuela de posgrado - Universidad Nacional Mayor de San Marcos \\ ORCID: 0000-0002-3018-9460
}

\author{
Katherin Santillán Guzmán \\ katherin.santillan@upeu.edu.pe \\ Escuela de Administración y Negocios Internacionales - Universidad Peruana Unión \\ ORCID: 0000-0002-6623-7727
}

Jorge Washington Saavedra Sandoval jwashingtonsaavedrasandoval@gmail.com Escuela de posgrado - Universidad Nacional Enrique Guzmán y Valle ORCID: 0000-0001-9178-5007

\begin{abstract}
RESUMEN
El presente estudio titulado: Liderazgo del director y motivación docente 2021, se realizó con el objetivo de determinar la relación entre el liderazgo del director y la motivación en los docentes de la I.E. N $\mathrm{N}^{\circ}$. 0006-Aplicación de Juanjuí, 2021; se utilizó el diseño correlacional; con una muestra de 40 trabajadores, seleccionados de manera no probabilística; se aplicó dos cuestionarios, para medir el liderazgo del director y la motivación en los docentes; validados mediante Juicio de Expertos, obteniéndose una alta confiablidad según el Alfa de Cronbach. Para el análisis de la información, se empleó técnicas estadísticas descriptivas y el coeficiente de correlación de Spearman para establecer el grado de correlación. Los resultados descriptivos revelan que el $45 \%$ de los docentes encuestados considera que el director tiene un liderazgo bueno y el 55\% considera que tiene un liderazgo aceptable; mientras que el $60 \%$ de los docentes encuestados revela una motivación media y el $40 \%$ revela una motivación alta. Por eso se concluye que existe una relación directa y significativa entre el Liderazgo del director y su relación con la motivación en los docentes de la I.E. Aplicación de Juanjuí, 2021; es decir, el buen liderazgo del director incide en la motivación de los docentes.
\end{abstract}

Palabras clave / Descriptores: liderazgo, motivación docente, gestión educativa, desempeño docente

\begin{abstract}
The present study entitled: Leadership of the director and teacher motivation 2021, was carried out with the objective of determining the relationship between the leadership of the director and the motivation in the teachers of the I.E. No. 0006-Application of Juanjuí, 2021; correlational design was used; with a sample of 40 workers, selected in a nonprobabilistic way; two questionnaires were applied to measure the leadership of the principal and the motivation of the teachers; validated by Expert Judgment, obtaining a high reliability according to Cronbach's Alpha. For the analysis of the information, descriptive statistical techniques and Spearman's correlation coefficient were used to establish the degree of correlation. The descriptive results reveal that $45 \%$ of the teachers surveyed consider that the director has good leadership and 55\% consider that he has acceptable leadership; while $60 \%$ of the teachers surveyed reveal a medium motivation and $40 \%$ reveal a high motivation. That is why it is concluded that there is a direct and significant relationship between the Director's Leadership and its relationship with the motivation in the teachers of the I.E. Application of Juanjuí, 2021; that is, the good leadership of the director affects the motivation of teachers.

Keywords / Descriptors: leadership, teacher motivation, educational management, teacher performance
\end{abstract}

\section{RESUMO}

O presente estudo intitulado: Liderança do diretor e motivação do professor 2021, foi realizado com o objetivo de determinar a relação entre a liderança do diretor e a motivação nos professores do I.E. Número 0006-Aplicación de Juanjuí, 2021; foi usado o desenho correlacional; com uma amostra de 40 trabalhadores, selecionados de forma não probabilística; foram aplicados dois questionários para medir a liderança do diretor e a motivação dos professores; validado por Expert Judgment, obtendo uma alta confiabilidade de acordo com o Alfa de Cronbach. Para a análise das informações, foram utilizadas técnicas de estatística descritiva e coeficiente de correlação de Spearman para estabelecer o grau de correlação. Os resultados descritivos revelam que $45 \%$ dos professores inquiridos consideram que o diretor 
tem uma boa liderança e 55\% consideram que tem uma liderança aceitável; enquanto $60 \%$ dos professores inquiridos revelam uma motivação média e $40 \%$ revelam uma motivação elevada. Por isso conclui-se que existe uma relação direta e significativa entre a Liderança do Diretor e a sua relação com a motivação nos docentes do I.E. Aplicação de Juanjuí, 2021; ou seja, a boa liderança do diretor afeta a motivação dos professores.

Palavras-chave / Descritores: liderança, motivação do professor, gestão educacional, desempenho do professor

\section{INTRODUCCIÓN}

La Institución Educativa de Aplicación Nº006 de Juanjuí, es una de las instituciones de educación básica de esta ciudad, el mismo que cuenta con los niveles de Educación Inicial, Primaria y Secundaria, los cuales funcionan de manera integrada. La ciudad de Juanjuí, es la capital de la província de Mariscal Cáceres; una de las 10 provincias que conforman la Región San Martín; esta región está ubicada, en la parte nor oriental de la Amazonía del Perú.

Como docentes sabemos que en la Institución Educativa de Aplicación, y en las demás instituciones de esta ciudad, se perdieron muchos años valiosos en la búsqueda de alcanzar la calidad educativa que todos deseamos, no sólo porque la Unidad de Gestión Educativa Local de Mariscal Cáceres- UGEL (órgano desconcentrado del Ministerio de Educación), encargaba el cargo de Director de la mencionada institución, a un docente diferente cada año, sino porque no se tomaba en cuenta si el Director encargado estaba debidamente capacitado y poseía un mínimo de liderazgo para dirigir la institución; teniendo como resultados negativos, el estancamiento en el desarrollo institucional y el escaso avance en el logro de la calidad educativa. Esta situación analizada, evidencia, que a lo largo de estos años de vida, de la Institución Educativa de Aplicación de Juanjuí, tanto las autoridades educativas, así como los Directores que estuvieron encargados, nunca le dieron ni la importancia ni mucho menos el valor al liderazgo del Director, porque si se hubiera entendido que el liderazgo del Director es fundamental para todos los logros institucionales, entonces actualmente otros serían los resultados, de ésta y de las demás instituciones educativas de nuestra localidade, región y país.

La nueva ley de carrera pública magisterial y otros dispositivos actuales vigentes exigen y condicionan de una manera imperativa el trabajo del director de las instituciones educativas, convirtiéndolo en un ente vertical, cumplidor administrativa y legal, deshumanizándolo y "obligándolo" a conductas más que educativas casi castrenses, para con sus colegas docentes.

El Ministerio de Educación del Perú, considera que uno de los grandes factores que contribuyen al logro de la calidad educativa y, por ende, a los logros de aprendizaje de los estudiantes, es el liderazgo del director. Para abordar el factor determinante del liderazgo del Director, ha elaborado el: "Marco del Buen Desempeño Directivo, que orienta y señala cuales son las características, desempeños y competencias que debe tener todo líder educativo, el mismo que para ejercer su cargo con responsabilidad y pertinencia es necesario que transite de un liderazgo inminentemente administrativo a un liderazgo pedagógico, que ponga el aprendizaje de los estudiantes en el centro del proceso pedagógico, que conduzca a la institución educativa a través del diálogo y la concertación, donde todos los miembros de la comunidad educativa compartan una idea y meta en común". Ministerio de Educación del Perú (2019) Manual del Buen Desempeño Directivo (MBDD). Perú, Lima. MINEDU.

El Ministerio de Educación del Perú, ha lanzado la política educativa "Todos podemos aprender, nadie se queda atrás", con el fin de garantizar la mejora de la calidad de los aprendizajes y que éstos lleguen a todos los estudiantes, para encontrar la calidad educativa que nos coloque en el escenario competitivo internacional como un país con ciudadanos capaces y competentes que contribuyan al desarrollo económico, social y cultural de nuestra patria. Para lograr este propósito ha diseñado una serie de programas, proyectos y estrategias que se están implementando progresivamente en las instituciones educativas a nivel nacional. Ministerio de Educación del Perú 
(2019) Currículo Nacional de la Educación. Perú, Lima, MINEDU. Sin embargo, es poco o nada lo que se está haciendo en la parte psicológica o emocional, tanto con los maestros, alumnos y padres de familia; en este sentido, no se ejerce un verdadero liderazgo, ni desde el Ministerio de Educación, mucho menos desde las unidades de Gestión Educativa; y poco son los Directores con predisposición, voluntad y capacidad para liderar a docentes, alumnos y padres de familia, que en buena cuenta, sería el aspecto que complementaría las políticas y todos los cambios planteados por la normatividad vigente del Ministerio de Educación.

En el ámbito regional, no se evidencia un verdadero liderazgo tanto a nivel de gobierno regional, así como a nivel de autoridades educativas y demás autoridades. No se asume y ni se trabaja para alcanzar la conducción de nuestra región con un debido liderazgo; por tanto las políticas de desarrollo regional, dentro de ellas, las políticas educativas no se logran a cabalidad o se retrasan en demasía, ya que para alcanzar cualquier propósito, más aún, el educativo, no basta con la entrega de materiales educativos, mejorar los locales escolares o supervisar a los maestros; la educación hoy en día, necesita ser conducida por verdaderos líderes, que entiendan el valor de su liderazgo, y que es fundamental dicho liderazgo, no sólo para tratar de alcanzar los logros de aprendizaje, sino también, para tratar de acortar las brechas que tenemos como país en vías de desarrollo, con respecto a los países del primer mundo.

A nivel local, sucede lo mismo, un ausentismo enorme de liderazgo, se puede observar a nuestras autoridades de diferentes niveles y responsabilidades, que carecen de absoluto liderazgo, no sólo porque, en la mayoría de los casos, no están debidamente preparados y capacitados para las responsabilidades asignadas; sino porque ni siquiera son conscientes de la gran responsabilidad que tienen al dirigir su institución hacia el logro de sus objetivos, dentro de los plazos y las urgencias que la sociedad en su conjunto así lo esperan. A nivel de la UGEL - Mariscal Cáceres, existe una cruda realidad respecto a los Directores de las instituciones educativas de educación básica regular, ya que de 150 plazas de Directores que existen en el presente año, sólo 17 de ellos fueron designados mediante un concurso y los 133 restantes fueron encargados, sin ninguna estabilidad, lo que significa además, que en el siguiente año podrían ser o no ratificados en el cargo; sin embargo lo que agrava aún más la situación es que de los 17 directores designados mediante un concurso el $50 \%$ de ellos no ejerce ningún liderazgo en su institución educativa ni en su comunidad. Unidad de Gestión Educativa Local de Mariscal Cáceres - Juanjuí (2019). Gestión Pedagógica.

A nivel de las instituciones educativas y específicamente a nivel de la Institución Educativa Aplicación $\mathrm{N}^{\circ} 0006$ de Juanjuí, somos testigos de que se perdieron muchos años valiosos en la búsqueda de alcanzar la calidad educativa que todos deseamos, no sólo porque la Unidad de Gestión Educativa Local de Mariscal Cáceres, encargaba el cargo de Director de la mencionada institución a un docente diferente cada año, sino porque no se tomaba en cuenta si el Director encargado estaba debidamente capacitado y poseía un mínimo de liderazgo para dirigir la institución; teniendo como resultados negativos, el estancamiento en el desarrollo institucional y el escaso avance en el logro de la calidad educativa total.

Esta situación analizada, evidencia, que a lo largo de estos años de vida, de la Institución Educativa Aplicación de Juanjuí, tanto las autoridades educativas, así como los Directores que estuvieron encargados, nunca le dieron ni la importancia ni mucho menos el valor al liderazgo del Director, porque si se hubiera entendido que el liderazgo del Director es fundamental para todos los logros institucionales, entonces actualmente otro serían los resultados institucionales, ya que en los años 98 Y 99, ya era una institución importante en la región; pero hubiéramos podido en todos estos años, pasar de ser una institución importante a una destacada.

Las limitaciones que han tenido, tanto las autoridades educativas, así como los Directores encargados que pasaron por la institución, de no darse cuenta, que para la conducción correcta de cualquier institución, más aún de una institución educativa, se necesita de un debido liderazgo; 
entendiendo que el director debe dar las iniciativas, debe organizar y dirigir la institución en todos los aspectos, debe ser el ejemplo a seguir por los docentes alumnos y padres de familia, debe demostrar antes de exigir, debe pregonar con el ejemplo; pero también debe saber motivar y estimular a sus docentes, alumnos y padres de familia por acciones destacadas, más aun reconociendo que un verdadero liderazgo es fundamental para lograr, de manera sustancial, la motivación de sus docentes y éstos logren en las aulas con sus alumnos, los propósitos educativos que el Perú de hoy necesita y así lo exige.

\section{FUNDAMENTO TEÓRICO}

En los dos últimos años, la Institución Educativa $\mathrm{N}^{\circ} 0006$ Aplicación de Juanjuí, ha obtenido algunos logros, en lo académico, en lo deportivo y en lo cultural; observándose también, una ligera mejora, respecto al liderazgo del Director y la motivación de los docentes" Reseña Histórica Bodas de Plata I.E. Aplicación Juanjuí (2016 p.3); sin embargo, y reconociendo que el avance respecto al liderazgo del Director aún es incipiente e insuficiente, nos hemos planteado realizar la presente investigación. A fin de contribuir con una mejor comprensión del estudio, presentamos algunos conceptos básicos:

\subsection{TEORÍAS RELACIONADAS AL TEMA:}

\subsubsection{Definiciones de liderazgo:}

- $\quad$ Rojas, A. y Gaspar, F. (2006), definen que: “el liderazgo es el arte de la conducción de seres humanos hacia el futuro" (p.18).

- Moyano, J., Bruque, S. Maqueira, JM., Fidalgo, FA. y Martínez, PJ. (2011), definen el liderazgo como: "La habilidad de los directivos de una organización para conseguir que los subordinados sigan las directrices marcadas por el directivo $\mathrm{o}$ líder, sin recurrir a acciones de coacción” (p.121).

- Fiedler, F. (1967), define el liderazgo como: "La relación entre el rendimiento organizacional y las actitudes del líder; es decir la forma como se cumplen las tareas y la relación del líder con sus subordinados" (p.16).

- Herzber, F. (1959), en su teoría de los dos factores define la motivación como: "El grado de satisfacción en el cargo y la naturaleza de las tareas que el individuo ejecuta" (p.62).

- Chiavenato, I. (2002), define el liderazgo como:” Un poder personal que permite a alguien influir en otros, por medio de las relaciones existentes. La influencia implica una transacción interpersonal, en la que un individuo actúa, para provocar o modificar un comportamiento" (p.336).

- $\quad$ Robins, SP. y Judge, TA. (2013), definen al liderazgo cómo: "La habilidad para influir en un grupo y dirigirlo hacia el logro de un objetivo o un conjunto de metas". (p.368).

- Hellriegel, D. y Slocum, J. (2009), establecen que liderazgo es: "El proceso de desarrollar ideas y una visión, de vivir conforme a los valores que apoyan esas ideas y esa visión, de influir en terceros para que los adopten en su propio comportamiento" (p.262). 


\subsubsection{Definiciones de motivación:}

- Moyano, J., Maqueira, JM., Fidalgo, FA. y Martínez, PJ. (2011), definen la motivación, como: "La habilidad para incitar a la acción de los subordinados, de tal forma que trabajen para conseguir los objetivos de la organización” (p.121).

- $\quad$ Robins, SP. Coulter, M. (2010), definen la motivación como: "los procesos que inciden en la intensidad, dirección y persistencia del esfuerzo, que realiza un individuo para la consecución de un objetivo" (p.175).

- $\quad$ Maslow, A. (1991), afirma que la motivación consiste en: "satisfacer las necesidades, fisiológicas, sociales, de seguridad, de estima y de autorrealización" (p. 393).

- $\quad$ Mcgregor, D. (1972), afirma que la motivación no es sino "el resultado de los factores, $x$ punto de vista negativo de la persona $y$, Y punto de vista positivo de las personas, el cual se debe aprovechar para maximizar su motivación" (p.94).

- Herzberg, F. (1959), en su teoría de los dos factores afirma que "La motivación es la satisfacción de los factores intrínsecos de la persona; en cambio la insatisfacción se relaciona con los factores extrínsecos de las personas” (p.395).

- Chiavenato, I. (2002), define la motivación como "un proceso que depende del curso, la intensidad y la persistencia de los esfuerzos de una persona para alcanzar determinados objetivos" (p.237).

\section{PROCEDIMIENTOS METODOLOGICOS}

El presente estudio es de tipo comparativo; sigue el diseño de investigación que corresponde al diseño correlacional, debido a que se propone determinar, el grado de relación entre las variables. Investigación orientada a la aplicación: Asimismo, el estudio es de tipo, no experimental, correlacional.

Este tipo de estudios tiene como finalidad conocer la relación o grado de asociación que exista entre dos o más conceptos, categorías o variables en una muestra o contexto en particular. En ocasio- nes sólo se analiza la relación entre dos variables, pero con frecuencia se ubican en el estudio vínculos entre tres, cuatro o más variables. Para evaluar el grado de asociación entre dos o más variables, en los estudios correlacionales pri- mero se mide cada una de éstas, y después se cuantifican, analizan y establecen las vinculaciones. Tales correlaciones se sustentan en hipótesis sometidas a prueba. Por ejemplo, un investigador que desee analizar la asociación entre la motivación laboral y la productividad individual, digamos, en varias empresas industriales con más de mil trabajadores de la ciudad de Santa Fe de Bogotá, Colombia, mediría la motivación y la productividad de cada persona y después analizaría si los trabajadores con mayor motivación son o no los más productivos (Sampieri 6ta. Edición. P.93).

Se optó por el tipo de investigación no experimental, correlacional, comparativo, porque el estudio, aplicará una encuesta a los docentes de dicha institución educativa, para dar respuesta a una realidad observable en las instituciones educativas de la ciudad de Juanjuí, es decir, establecer de manera científica, si existe relación entre el liderazgo del director y la motivación en los docentes de la I.E. Aplicación de Juanjuí, 2021. Asimismo, el estudio es de tipo, no experimental, correlacional. 


\subsection{TÉCNICAS E INSTRUMENTOS DE RECOLECCIÓN DE DATOS, VALIDEZ Y CONFIABILIDAD}

3.1.1. Técnicas: En el presente estúdio, se empleó como técnica la encuesta, que se aplicó a los 40 docentes de la I.E. Aplicación de Juanjuí, 2021 que fueron seleccionados, exceptuando a aquellos contratados en el último año, a los que estaban ocupando licencias por algunos meses y a aquellos delicados de salud.

3.1.2. Instrumentos: Para el desarrollo del trabajo de investigación, y recolección de los datos se empleó como instrumentos dos cuestionarios:

\section{- Cuestionario para medir el liderazgo del diretor:}

Su finalidad fue determinar el nivel de liderazgo del director. Además, el instrumento constó de 18 ítems, distribuidos en base a los indicadores. El encuestado tiene tres opciones de respuesta: 3: siempre, 2: a veces 1; nunca.

\section{- Cuestionario sobre motivación en los docentes:}

El propósito del presente instrumento fue medir el nivel de motivación en los docentes de la I.E. Aplicación de Juanjuí, 2021. El cuestionario tipo encuesta constó de 18 ítems distribuidos entre dos dimensiones. Las opciones de respuesta fueron: 3: Siempre 2: A

veces 1: nunca:

3.1.3. Validez y confiabilidad del instrumento: Los instrumentos de investigación empleados en este estudio, han sido validados mediante juicio de expertos; habiéndose obtenido una validez y confiabilidad alta. La confiabilidad de nuestros instrumentos se realizará con el Alfa de Cronbach $>0.70$

3.1.4. Métodos de análisis de datos: En el ordenamiento y procesamiento de datos se utilizó técnicas estadísticas descriptivas; para constituir y presentar los datos, se manipuló tablas y gráficas de barras; así como, las técnicas descriptivas; la frecuencia absoluta y porcentual; la media, máximo, mínimo, rango y la desviación estándar. Para establecer el grado de acercamiento y dirección de las variables, se utilizó el coeficiente de correlación de Pearson mediante la fórmula o estadístico $\rho$ viene dado por la expresión:

$$
\mathrm{r}=\frac{\mathrm{n} \sum \mathrm{xy}-\left(\sum \mathrm{x}\right)\left(\sum \mathrm{y}\right)}{\sqrt{\mathrm{n}\left(\sum \mathrm{x}^{2}\right)-\left(\sum \mathrm{x}\right)^{2} \cdot \sqrt{\mathrm{n}\left(\sum \mathrm{y}^{2}\right)-\left(\sum \mathrm{y}\right)^{2}}}}
$$

Asimismo, en el presente estudio, se contó con una participación informada, de los docentes de la institución, material de estudio, el cual se materializó mediante una autorización por parte de la dirección institucional, previa presentación e información detallada del proyecto de estudio. No hubo conflicto de valores alguno. El Anexo 1 evidencia este punto aquí descrito.

\section{RESULTADOS Y DISCUSIÓN}

\subsection{Resultados 1:}

En la tabla $\mathrm{N}^{\circ} 1$ se presentan los datos recolectados referidos al liderazgo del director y la motivación en los docentes; ambos recogidos mediante un cuestionario cuyo máximo puntaje fue de 54 puntos para cada variable de estudio. Ambos instrumentos fueron aplicados a los docentes de la 
I.E. Aplicación de Juanjuí, 2021. En tal sentido, se aprecia los puntajes obtenidos por cada uno de los sujetos; observándose un puntaje grupal de 1804 puntos, una media de 45, así como un puntaje máximo de 54 y un mínimo de 25 (29 puntos de rango) y una desviación estándar de 7.47 desviaciones respecto a la media, deduciéndose un aceptable y heterogéneo liderazgo del director. Asimismo, se observa los puntajes inherentes a la motivación en los docentes, notándose un puntaje grupal de 1842 puntos, una media de 46, así como un puntaje máximo de 54 y un mínimo de 26 puntos (28 puntos de rango), con una desviación estándar de 5, 9 desviaciones respecto a la media; lo cual indica un regular nivel de motivación.

Tabla N. 1 Puntajes obtenidos respecto al liderazgo del director y la motivación en los docentes.

\begin{tabular}{|c|c|c|c|c|c|c|}
\hline \multirow[b]{2}{*}{ N.. } & \multicolumn{3}{|c|}{ LIDERAZGO DEL DIRECTOR } & \multicolumn{3}{|c|}{ MOTIVACION EN LOS DOCENTES } \\
\hline & $\begin{array}{l}\text { INICIATIVA } \\
\text { DEL } \\
\text { DIRECTOR }\end{array}$ & $\begin{array}{l}\text { CUALIDADES } \\
\text { DEL } \\
\text { DIRECTOR }\end{array}$ & PUNTAJE & $\begin{array}{c}\text { ESTADO } \\
\text { EMOCIONAL }\end{array}$ & $\begin{array}{c}\text { ELOGIOS Y } \\
\text { RECOMPENSAS }\end{array}$ & PUNTAJE \\
\hline 1 & 25 & 26 & 51 & 26 & 24 & 50 \\
\hline 2 & 22 & 20 & 42 & 24 & 20 & 44 \\
\hline 3 & 22 & 25 & 47 & 24 & 25 & 49 \\
\hline 4 & 27 & 27 & 54 & 26 & 25 & 51 \\
\hline 5 & 16 & 15 & 31 & 23 & 19 & 42 \\
\hline 6 & 24 & 25 & 49 & 24 & 26 & 50 \\
\hline 7 & 27 & 23 & 50 & 23 & 27 & 50 \\
\hline 8 & 16 & 19 & 35 & 23 & 18 & 41 \\
\hline 9 & 24 & 26 & 50 & 23 & 26 & 49 \\
\hline 10 & 24 & 25 & 49 & 24 & 24 & 48 \\
\hline 11 & 25 & 25 & 50 & 25 & 25 & 50 \\
\hline 12 & 25 & 23 & 48 & 25 & 23 & 48 \\
\hline 13 & 15 & 10 & 25 & 21 & 19 & 40 \\
\hline$\ldots$ & $\ldots$ & $\ldots$ & $\ldots$ & $\ldots$ & $\ldots$ & $\ldots$ \\
\hline$\ldots$ & $\ldots$ & $\cdots$ & $\cdots$ & $\ldots$ & $\cdots$ & $\cdots$ \\
\hline$\ldots$ & $\ldots$ & $\ldots$ & $\ldots$ & $\ldots$ & $\ldots$ & $\ldots$ \\
\hline 33 & 27 & 25 & 52 & 25 & 26 & 51 \\
\hline 34 & 23 & 23 & 46 & 22 & 12 & 34 \\
\hline 35 & 27 & 27 & 54 & 27 & 27 & 54 \\
\hline 36 & 18 & 20 & 38 & 22 & 19 & 41 \\
\hline 37 & 19 & 19 & 38 & 23 & 19 & 42 \\
\hline 38 & 27 & 27 & 54 & 26 & 21 & 47 \\
\hline 39 & 24 & 23 & 47 & 24 & 24 & 48 \\
\hline 40 & 27 & 25 & 52 & 27 & 25 & 52 \\
\hline SUMA & 908 & 896 & 1804 & 970 & 872 & 1842 \\
\hline
\end{tabular}




\begin{tabular}{|c|c|c|c|c|c|c|}
\hline MEDIA & 22.7 & 22.4 & 45.1 & 24.25 & 21.8 & 46.05 \\
\hline DS & 3.94 & 4.03 & 7.47 & 2.15 & 4.32 & 5.9 \\
\hline RANGO & 15 & 17 & 29 & 12 & 18 & 28 \\
\hline
\end{tabular}

Fuente: Datos obtenidos de los instrumentos aplicados a los docentes de la Institución Educativa No 0006-Aplicación de Juanjuí, para medir el liderazgo del director y la motivación en los docentes.

\subsection{Resultados 2:}

En la tabla N. ${ }^{\circ}$ 2, se observa un resumen de las medidas estadísticas descriptivas centrales y de dispersión de los 40 sujetos muestrales; en la que se aprecia las medias, las desviaciones estándar y el coeficiente de variación para cada una de las variables y sus respectivas dimensiones. Notándose una dispersión pequeña de 3.94 desviaciones respecto a la media $(22,7)$ de iniciativa del director, incrementándose ligeramente a 4.03 desviaciones respecto a la media $(22,4)$ de cualidades del director, acumulando una dispersión de 7.47 desviaciones respecto a la media $(45,1)$ de liderazgo del director, el cual es bastante favorable para la motivación docente; es decir el 16\% de los datos están dispersados entre sí y respecto a la media.

Asimismo, se visualiza una dispersión relativamente baja de 2.15 desviaciones respecto a la media $(24,25)$ en su estado emocional y un notable incremento a 4.32 desviaciones respecto a la media (21.8) de elogios y recompensas; acumulando una dispersión de 5.9 respecto a la media $(46,6)$ de su motivación docente; la misma que es muy aceptable; es decir, el 12\% de los datos están esparcidos entre sí y respecto a la media.

Tabla N. 2 Resumen de medidas estadísticas descriptivas inherentes a las variables de estudio.

\begin{tabular}{|c|c|c|c|c|}
\hline $\begin{array}{c}\text { VARIABLES Y } \\
\text { SUBVARIABLES }\end{array}$ & Media & Desviación típica & $\begin{array}{c}\text { Coeficiente de } \\
\text { variación }\end{array}$ & $\mathbf{N}^{\circ}$ \\
\hline Iniciativa del director & 22.7 & 3.94 & 0.17 & 40 \\
\hline Cualidades del director & 22.4 & 4.03 & 0.17 & $\mathbf{4 0}$ \\
\hline Liderazgo del director & 45.1 & 7.47 & 0.16 & 40 \\
\hline Estado emocional & 24.25 & 2.15 & 0.08 & 40 \\
\hline Elogios y recompensas & 21.8 & 4.32 & 0.19 & $\mathbf{4 0}$ \\
\hline Motivación docente & 46.05 & 5.9 & 0.12 & \\
\hline
\end{tabular}

Fuente: Elaboración propia de los autores.

\subsection{Resultado 3:}

En la tabla $\mathrm{N}^{\mathrm{o}} 3$ se aprecia el nivel de liderazgo del director, a partir de la percepción de los docentes de la I.E. Aplicación de Juanjuí, 2021, en función de la escala de medición cualitativa y cuantitativa; distinguiéndose que el $45 \%$ de los docentes (18) afirman que es bueno el liderazgo del director; es decir, sus puntuaciones fluctúan entre 49 y 54 puntos; el $55 \%$ restante de los docentes (22) dan cuenta de un liderazgo a nivel aceptable del director; ya que sus puntuaciones oscilan entre 25 y 45 puntos respectivamente. 
Tabla No 3 Nivel de liderazgo del director de la I.E. Aplicación de Juanjuí, 2016.

\begin{tabular}{|c|c|c|c|}
\hline \multicolumn{2}{|c|}{ ESCALA DE VALORACIÓN } & f & \% \\
\hline BUENO & $49-54$ & 18 & 55 \\
\hline ACEPTABLE & $25-45$ & 22 & 00 \\
\hline DEFICIENTE & $18-24$ & 00 & $\mathbf{1 0 0}$ \\
\hline \multicolumn{2}{|c|}{ TOTAL } & 40 & \\
\hline
\end{tabular}

Fuente: Elaboración propia de los autores.

\section{CONCLUSIONES}

Existe una relación alta con dirección positiva, entre el liderazgo del director y su motivación en los docentes de la I.E. Aplicación de Juanjuí, provincia de Mariscal Cáceres, durante el periodo 2021, ya que el resultado de la correlación es de 0,73 ; lo cual significa que los directores que ejercen un elevado nivel de liderazgo manifestadas a través de sus iniciativas y cualidades están relacionados positivamente con la motivación en los docentes.

El nivel de liderazgo del director de la I.E. Aplicación de Juanjuí, provincia de Mariscal Cáceres, durante el periodo 2021 es aceptable; es decir, el director deja evidenciar de forma regular, a través de su accionar diario, cualidades e iniciativas positivas, los cuales gozan de la aprobación de la mayoría de los docentes.

El nivel de motivación en los docentes de la I.E. Aplicación de Juanjuí, provincia de Mariscal Cáceres durante el periodo 2021, es media; es decir, los docentes evidencian regular motivación en relación con sus estados emocionales y con el reconocimiento, elogios y recompensas recibidas por parte del director.

En el presente estudio, se encontraron algunas limitaciones, más que de tipo teóricas y metodológicas, las limitantantes fueron, especialmente en el aspecto logístico y de los medios tecnológicos, al no contar en nuestra zona, con las tecnológías más avanzadas y la dificultad, por la mala calidad de los servicios de internet, energía electrica y otros servicios.

A partir de los resultados del presente estudio, sugerimos investigaciones, sobre los resultados académicos, en colegios dirigidos por directores debidamente capacitados en liderazgo y motivación; asimismo sobre, cuál es el nivel de influencia en el rendimiento académico de los estudiantes, el desempeño laboral de un docente debidamente capacitado y motivado.

\section{REFERENCIAS}

Andrew, J. (2008). Relaciones Humanas: Comportamiento humano en el Trabajo (9a ed.) México, DF.

Arruada, J. (1982). Didáctica y Práctica de la Enseñanza. (1 $1^{\mathrm{a}}$ ed.) Colombia, Bogotá.

Carrasco, S. (2002). Gestión Educativa y Calidad de Formación Profesional en la Facultad de Educación. UNMSM.

Castillo, V. (2010). "Relación del liderazgo de la directora y el desempeño laboral de los docentes de la I.E.I. $N^{\circ} 87$ Callao”. (Tesis para optar el grado de Magister en Gestión de la Educación). 
Chanducas, L. (2014). "Liderazgo transformacional de los directores y la satisfacción laboral de los docentes de la Asociación Educativa Adventista Nor Oriental”. (Tesis para optar el grado académico de Magister en Educación, con mención en Administración Educativa).

Chiavenato, I. (2002). Comportamiento Organizacional. (2ª ed.) México, DF.

Chiavenato, I. (2009). Gestión del Talento Humano. (3ª ed.) México, DF.

Chiroque, S. (2006). Evaluación del Desempeño Docente. ( $1^{\mathrm{a}} \mathrm{ed}$.) Perú, Lima.

De La Cruz, L. (2008). Evaluación del Desempeño Profesional Docente. (1ª ed.) Perú, Lima.

Escamilla, S. (2008). "Estudio del director escolar y sus necesidades de formación para un desempeño profesional óptimo en el campo de la educación básica del estado de Nuevo León en México” (Tesis Doctoral).

Fiedler, F. (1977). Teoría de la Eficiencia del Liderazgo. (2 $2^{\mathrm{a}}$ ed.) Estados Unidos, Nueva York

Gago, F. (2002). Modelo para la evaluación del desempeño docente en la función docencia universitaria ( $1^{\mathrm{a}}$ ed.) España, Madrid.

Gómez, L. Balkin, D. y Cardy, R (2005). Gestión de Recursos Humanos. (5ª ed.) España, Madrid.

Gutiérrez, H. (2010). Calidad Total y Productividad. (3ª ed.) México, DF.

Hellriegel, D. Jackson, S. Slocum, J. (2009). Administración un Enfoque Basado en Competencias. (11ª ed.) México, DF.

Hellriegel, D. y Slocum, J. (2009). Comportamiento Organizacional. (12 ed.) México, DF.

Hernández, R. Fernández, C. Baptista, P. (2010). Metodología de la Investigación. (11 a ed.) México, DF.

Herzber, F. (1959). Motivación al Trabajo. (1 ${ }^{\mathrm{a}}$ ed.) Estados Unidos, Utah.

Jiménez, C. (2014). "Relación entre el liderazgo transformacional de los directores y la motivación hacia el trabajo y el desempeño de docentes de una universidad privada de Bogotá - Colombia” (Tesis para optar el grado de Magister en Psicología).

Koontz, H y Weihrich, H. (1998). Administración: Una Perspectiva Global. (11 a ed.) México, DF.

Koontz, H. y Weihrich, H. (2013) Elementos de Administración. (8ª ed.) México, DF.

Loza, E. (2014). “Liderazgo y compromiso organizacional en los docentes de la I.E.P. Simón Bolivar de la ciudad de Tarapoto”. (Tesis para optar el grado académico de Maestro en Psicología, con mención en Psicología Organizacional).

Maslow, A. (1991). Motivación y Personalidad. (3ª ed.). España, Madrid.

McGregor, D. (1972). Mando y Motivación. (2a ed.) México, DF.

Ministerio de Educación del Perú (2008). Catálogo nacional de títulos y certificaciones. Perú, Lima, MINEDU.

Ministerio de Educación del Perú (2016). Currículo Nacional de la Educación. Perú, Lima, MINEDU.

Ministerio de Educación del Perú (2016). Manual del Buen Desempeño Directivo (MBDD). Perú, Lima, MINEDU.

Mondy, W. (2000). Actualizaciones para el Management y el Desarrollo Organizacional. (1 ${ }^{\text {a }}$ ed.) Chile, Santiago.

Morán, P. (2012). La evaluación cualitativa en los procesos y prácticas del trabajo en el aula, México. Editorial IISUE.

Moyano, J., Maqueira, JM., Fidalgo, FA. y Martínez, PJ. (2011) Administración de Empresas. (1 ${ }^{\text {a }}$ ed.) España, Madrid.

Puchol, L. (2003). Dirección y Gestión de los Recursos Humanos. (5 $5^{\mathrm{a}}$ ed.) España, Madrid.

Reseña Histórica (2016). Bodas de Plata I.E. Aplicación. Perú, San Martín, Mariscal Cáceres, Juanjuí.

Rincón, JC. (2005). Liderazgo directivo y desempeño de los docentes del Valle del Chumbao de la provincia de Andahuaylas - Apurímac.

Rivera, M. (2008). "El liderazgo y la gestión de los directores en las unidades de gestión educativa local de la región Junín” (Tesis Doctoral)

Robins, SP. Coulter, M. (2010). Administración (10ª ed.) México, DF.

Robins, SP. y Judge, TA. (2013). Comportamiento Organizacional (15 a ed.) México, DF.

Rojas, A. y Gaspar, F. (2006). Bases del Liderazgo en Educación (1 ${ }^{\mathrm{a}}$ ed.) ORELAC/UNESCO. Chile, Santiago

Rubio, P. (2007). Introducción a la Gestión Empresarial (1 ${ }^{\mathrm{a}}$ ed.) España, Madrid.

Rugarcía, A. (1994) El Compromiso Ético del Docente. (1 ${ }^{\mathrm{a}}$ ed.) México, Puebla.

Salas, M. (2011). La Evaluación del Desempeño de los Docentes. (1 $1^{\mathrm{a}}$ ed.) México, DF.

Sorados, M. (2010). Influencia del liderazgo en la calidad de la gestión educativa (Tesis para optar el grado de Magíster en Educación con Mención en Gestión de la Educación).

Stephen, P. Robbins. Timothy, A. Judge (2015). Comportamiento Organizacional. (15 a ed.) México, DF.

Stiggins, R. y Duke, D. (1998). Evaluación del Desempeño Docente. Estados Unidos, Portland, Oregon.

Tarazona, O. (2008). Así es mi Tierra. San Martín, Mariscal Cáceres, Juanjuí. 
Thieme, C. (2005). "El liderazgo y la eficiencia en la educación primaria - El Caso Chile” (Tesis Doctoral). UGEL-Mariscal Cáceres-Juanjuí (2016). Área: Gestión Pedagógica.

Valdés, H. (2000). Calidad de la Educación Básica y su Evaluación. (1ª ed.) España, Madrid.

Valdés, H. (2004). Reflexiones sobre la Calidad Educativa (1 ${ }^{\mathrm{a}}$ ed.) México, DF.

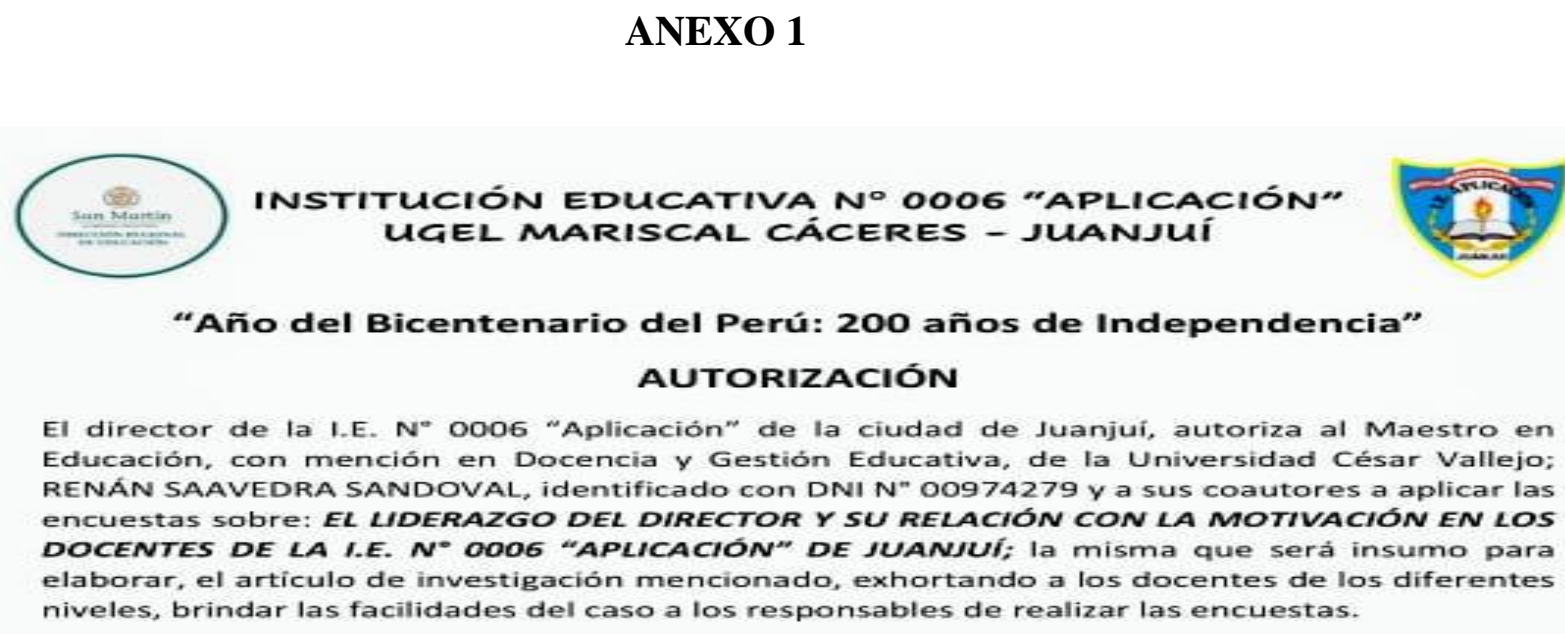

Atentamente.

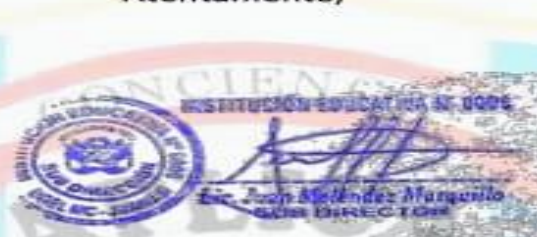

